\title{
Dual energy CT is useful for the prediction of mesenteric and lateral pelvic lymph node metastasis in rectal cancer
}

\author{
KENTARO SATO $^{1}$, HAJIME MOROHASHI ${ }^{1}$, FUMIYASU TSUSHIMA ${ }^{2}$, YOSHIYUKI SAKAMOTO ${ }^{1}$, \\ TAKUYA MIURA $^{1}$, HIROMASA FUJTAA ${ }^{2}$, KOTARO UMEMURA ${ }^{1}$, TAKAHIRO SUZUKI ${ }^{1}$, SATORU TSURUTA ${ }^{1}$, \\ RYOTO KURA $^{1}$, SHUICHI ONO ${ }^{2}$, MASAHIKO AOKI ${ }^{2}$ and KENICHI HAKAMADA ${ }^{1}$ \\ Departments of ${ }^{1}$ Gastroenterological Surgery and ${ }^{2}$ Radiation and Oncology, \\ Hirosaki University Graduate School of Medicine, Hirosaki, Aomori 036-8562, Japan
}

Received September 7, 2018; Accepted March 20, 2019

DOI: $10.3892 / \mathrm{mco} .2019 .1834$

\begin{abstract}
The aim of the present retrospective study was to investigate the predictability of dual-energy computed tomography (DECT) for pararectal lymph node (PRLN) metastasis and lateral pelvic lymph node (LPLN) metastasis in rectal cancer (RC). The present study involved 44 patients with RC who were examined by DECT and then underwent surgery between May 2015 and September 2017. LPLN dissection was performed in 24 patients. The normalized iodine concentration (nIC), the ratio of iodine concentration in the lymph node (LN) to that in the common iliac artery on DECT, of the largest PRLN and LPLN was calculated, and the association between LN metastasis and nIC was analyzed. The median nIC value for PRLNs was significantly lower in PRLN metastasis-positive cases compared with PRLN metastasis-negative cases in the arterial phase [0.18 vs. $0.25 ; \mathrm{P}=0.01$; cut-off, 0.24; area under the curve (AUC), 0.733] and portal phase ( 0.47 vs. $0.61 ; \mathrm{P}=0.03$; cut-off, 0.59 ; AUC, 0.701 ). A significant difference was not identified between the median maximum short axis diameter of PRLNs in PRLN metastasis-positive and metastasis-negative cases ( 7.6 vs. $6.4 \mathrm{~mm} ; \mathrm{P}=0.33$ ). The nIC for LPLNs was not significantly different between LPLN metastasis-positive and metastasis-negative cases in the arterial phase ( 0.15 vs. $0.21 ; \mathrm{P}=0.19)$; but was significantly lower
\end{abstract}

Correspondence to: Professor Kenichi Hakamada, Department of Gastroenterological Surgery, Hirosaki University Graduate School of Medicine, 5 Zaifu-cho, Hirosaki, Aomori 036-8562, Japan

E-mail: hakamada@hirosaki-u.ac.jp

Abbreviations: DECT, dual energy computed tomography; RC, rectal cancer; LN, lymph node; CIA, common iliac artery; PRLN, pararectal lymph node; LPLN, lateral pelvic lymph node; nIC, normalized iodine concentration; NAC, neoadjuvant chemotherapy; NACRT, neoadjuvant chemoradiotherapy; AP, arterial phase; PP, portal venous phase; ROI, region of interest; PPV, positive predictive value; NPV, negative predictive value; AUC, area under the curve

Key words: DECT, RC, LPLN, PRLN, lymph node metastasis, iodine concentration in LPLN metastasis-positive cases compared with LPLN metastasis-negative cases in the portal phase $(0.29$ vs. 0.56 ; $\mathrm{P}=0.04$; cut-off, 0.29; AUC, 0.877). The maximum short axis diameter of LPLNs was significantly larger in metastasis-positive cases compared with LPLN metastasis-negative cases (9.1 vs. $4.8 \mathrm{~mm}$; $\mathrm{P}=0.03$; cut-off, $7.0 \mathrm{~mm}$; AUC, 0.912). In conclusion, the nIC was identified to be significantly lower in metastasis-positive cases, which may be useful for the prediction of PRLN and LPLN metastases. A combination of size-based diagnosis and DECT may increase the accuracy of preoperative diagnosis.

\section{Introduction}

In rectal cancer $(\mathrm{RC})$, lymph node (LN) metastasis is a poor prognostic factor $(1,2)$ and improvement of outcomes using neoadjuvant chemotherapy (NAC) or neoadjuvant chemoradiotherapy (NACRT) has previously been reported $(3,4)$. Appropriate introduction of preoperative treatment requires accurate prediction of lymph node metastasis prior to surgery. At present, size-based diagnosis using the maximum short axis diameter of LNs on computed tomography (CT) or magnetic resonance imaging (MRI) is predominantly used to predict metastasis $(5,6)$. However, diagnostic accuracy of size-based diagnosis is unsatisfactory. Ogawa et al (6) reported diagnostic accuracy of short axis diameter for pararectal LN (PRLN) was $63.7 \%$ (cutoff; $5 \mathrm{~mm}$ ) and for laterally pelvic lymph node (LPLN) was $77.6 \%$ (cutoff; $5 \mathrm{~mm}$ ) in MRI findings. These accuracies were not enough to introduce preoperative therapy appropriately, so studies focusing on the 'quality' of LNs have recently been tried to improve the diagnostic accuracy. Previously, prediction of PRLN metastasis in RC using dual-energy CT (DECT) has been reported (7). By contrast, to the best of our knowledge, prediction of LPLN metastasis in low RC using DECT has not been previously reported. LPLN dissection has been performed for locally advanced low RC in Japan, which has been demonstrated to reduce the rate of local recurrence (8). However, LPLN dissection can cause complications, including increased blood loss, postoperative dysuria and sexual dysfunction (8-10); therefore, selection of patients is necessary. However, the diagnostic reliability of size-based diagnosis for LPLN metastasis is unsatisfactory. In a JCOG0212 study (8), the pathological 
LPLN metastasis-positive rate was only $7 \%$ in patients with a maximum short axis diameter $\leq 10 \mathrm{~mm}$ on preoperative MRI, and LPLN dissection was not required for $>90 \%$ of the patients. These findings suggest that size-based diagnosis alone is insufficient for the prediction of LPLN metastasis and a different approach is required for the selection of patients. The aim of the present study was to investigate the predictability of DECT for PRLN and LPLN metastasis in RC.

\section{Patients and methods}

Patients. The current study involved 44 patients with RC who were examined preoperatively using DECT and then underwent surgery at our department between May 2015 and September 2017. During these periods, DECT was used as the routine preoperative CT examination for clinical staging. Patients who underwent preoperative chemotherapy $(n=25)$ were examined by DECT prior to preoperative therapy. Samples examined by DECT were analyzed retrospectively. The present study was approved by the Human Research Ethics Committee of the Hirosaki University Graduate School of Medicine (Aomori, Japan; reference no. 2018-1047). The clinical stage was judged using the 8th edition of the Japanese Classification of Colorectal Carcinoma (11). LPLN dissection was performed in 24 patients with lower RC in whom the clinical invasion depth of tumor was T3 or deeper (deeper than the muscularis propria), with the lower margin present on the anal side of the peritoneal reflection. LPLN dissection was performed bilaterally. The histopathological evaluation of LNs were performed retrospectively from the results of routine pathological diagnosis by pathologists.

Dual-energy CT technique. DECT imaging was performed using a Discovery 750 HD system (GE Healthcare, Chicago, IL, USA) with a fast kilovoltage switching method, as previously reported by Aoki et al (12), with a few adjustments. Briefly, a non-ionic contrast medium dose of $600 \mathrm{mgI} / \mathrm{kg}$ body weight, with an iodine content of $300 \mathrm{mgI} / \mathrm{ml}$ (Omnipaque 300; Daiichi-Sankyo Co. Ltd., Japan), was administered. The total amount of contrast medium was intravenously injected within $30 \mathrm{sec}$, and scanning of the arterial phase (AP) and portal venous phase (PP) began 35 and $70 \mathrm{sec}$ after initiating the injection of contrast medium. The thickness of the slice analyzed was $1.25 \mathrm{~mm}$.

Imaging analysis. DECT images were transferred to a workstation (Advantage workstation 4.6; GE Healthcare) for analysis. One surgeon (KS) and one medical student (RK) analyzed the images. Using iodine overlay images, the iodine concentration of LNs $\left(\mathrm{IC}_{\mathrm{LN}}\right)$ was measured by a circular region of interest (ROI) using the extracted maximum short axis diameter of the PRLN and LPLN in the AP and PP (Fig. 1A and B). The iodine concentration of the common iliac artery $\left(\mathrm{IC}_{\mathrm{CIA}}\right)$ was measured for the right common iliac artery in the AP and PP (Fig. 1C). The normalized iodine concentration (nIC) value was calculated by the following formula, $\mathrm{nIC}=\mathrm{IC}_{\mathrm{LN}}(\mathrm{mg} / \mathrm{ml}) / \mathrm{IC}_{\mathrm{CIA}}$ $(\mathrm{mg} / \mathrm{ml})$, as previously described by Liu et al (7).

Selection of evaluated LNs and radiological-histopathological comparison. The largest PRLN and the largest LPLN inside the dissection area were selected to evaluate nIC value by DECT. LPLN dissection was performed bilaterally, and larger side
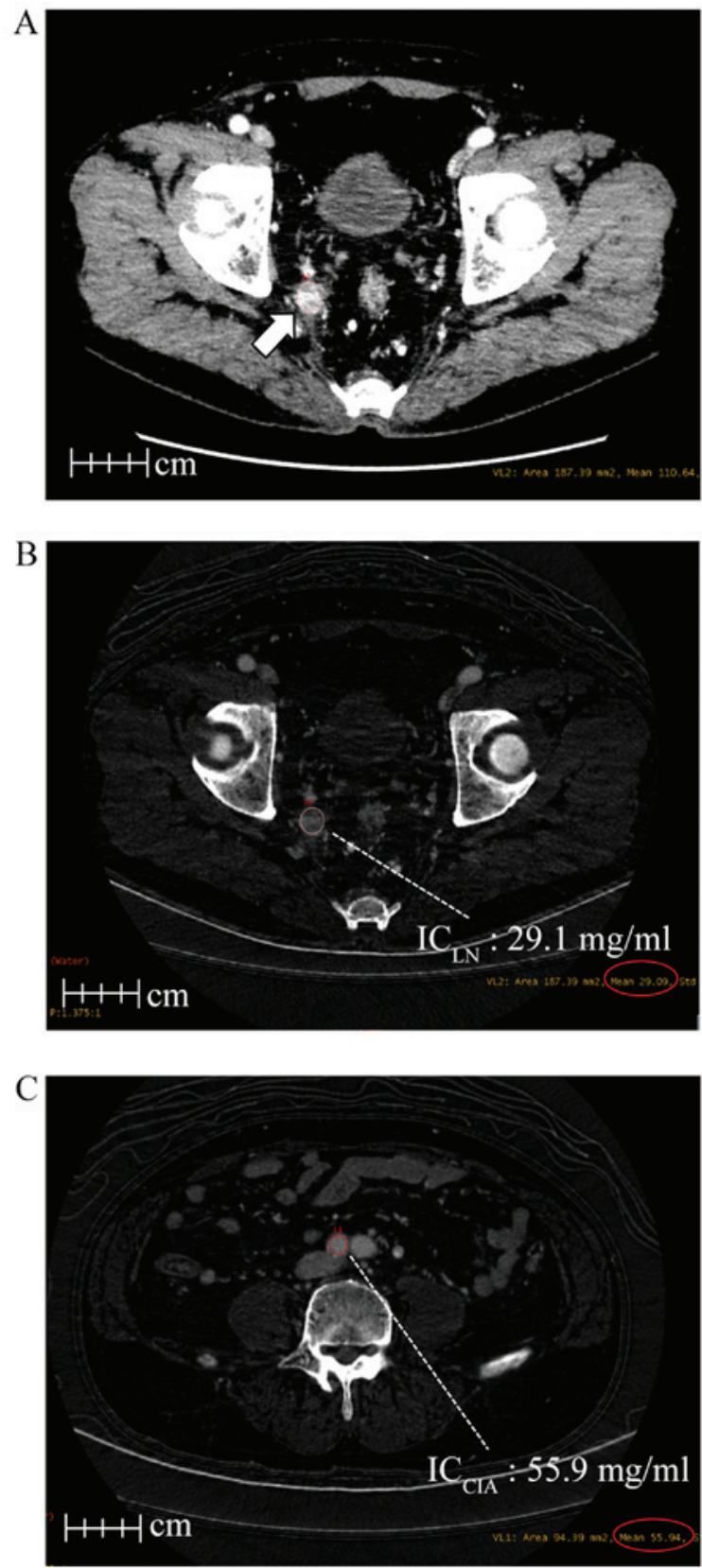

Figure 1. Example of the calculation for nIC by DECT. (A) An axial computed tomography image demonstrated the extracted maximum short axis diameter LPLN in the portal venous phase (white arrowhead). (B and C) Iodine overlay image. Each circle indicates the (B) lesion of interest of LPLN and (C) right CIA. In this case, the $\mathrm{IC}_{\mathrm{LN}}$ was $29.1 \mathrm{mg} / \mathrm{ml}$ and the $\mathrm{IC}_{\mathrm{CIA}}$ was $55.9 \mathrm{mg} / \mathrm{ml}$. The $\mathrm{nIC}$ was $0.52 \mathrm{mg} / \mathrm{ml}\left(\mathrm{nIC}=\mathrm{IC}_{\mathrm{LN}} / \mathrm{IC}_{\mathrm{CIA}}\right)$. LPLN, lateral pelvic lymph node; CIA, common iliac artery; $\mathrm{IC}_{\mathrm{LN}}$, iodine concentration of lymph node; $\mathrm{IC}_{\mathrm{CIA}}$, iodine concentration of CIA; nIC, normalized iodine concentration.

LPLN was selected for evaluation. Patients with a maximum short axis diameter $\leq 3 \mathrm{~mm}$ LNs were excluded because of difficulty in extracting the ROI. Following the exclusion, the nIC values for PRLNs and LPLNs were calculated in 43/44 and 22/24 patients, respectively. Pathological PRLN and LPLN metastasis were evaluated from the results of the routine pathological diagnosis for the staging of RC.

Methods of radiological-histopathological comparison was following; when pathological metastatic-positive LNs (at least $\geq 1$ ) existed in the pathologically-examined LNs, radiologically-selected LNs by DECT were metastasis-positive. 
When there were no pathologically-metastatic LNs, radiologically-selected LNs by DECT were metastasis-negative. Radiological-histopathological comparison for LPLN was performed using only one side selected for calculating nIC by DECT. For example, when the nIC was calculated from the right side LPLN by DECT, pathological evaluation was performed using only right side LPLNs.

Statistical analysis. Associations of nIC and short axis diameter for PRLNs and LPLNs with metastasis were investigated statistically. Cut-off values for these associations were determined using receiver operating curve (ROC) analysis, and the area under the curve (AUC), sensitivity, specificity, positive predictive value (PPV), negative predictive value (NPV) and accuracy at the cut-off were determined. Statistical analysis was performed by Mann-Whitney U-test and $\chi^{2}$ test. $\mathrm{P}<0.05$ was considered to indicate a statistically significant difference. These analyses were performed using Easy R software (13).

\section{Results}

Clinical characteristics. The clinical characteristics of the patients are presented in Table I. PRLN metastasis was detected in $34.1 \%$ of the 44 patients. LPLN dissection was performed in 24 patients $(54.5 \%)$ and LPLN metastasis was detected in 3 of these patients $(12.5 \%)$.

Associations of size and nIC of PRLNs for PRLN metastasis. The associations of the maximum short axis diameter of PRLNs and nIC in the AP and PP in cases with and without PRLN metastasis are presented in Table II. The median maximum short axis diameter of PRLNs was insignificantly different between PRLN metastasis-positive and metastasis-negative cases ( 7.6 vs. $6.4 \mathrm{~mm} ; \mathrm{P}=0.33$ ). The median nIC of the maximum-size PRLN was significantly lower in the PRLN metastasis-positive cases compared with the PRLN metastasis-negative cases in the AP $(0.18$ vs. $0.25 ; \mathrm{P}=0.01)$ and in the PP (0.47 vs. $0.61 ; \mathrm{P}=0.03)$. The cut-off values of $\mathrm{nIC}$ for PRLNs in ROC analysis were 0.24 (AUC, 0.733) and 0.59 (AUC, 0.701) in the AP and PP, respectively (Fig. 2A and B), and these cut-off values provided a sensitivity, specificity, PPV, NPV and accuracy of $86.7,51.9,48.1,83.3$, and $62.8 \%$ for the AP, respectively, and 80, 55.6, 48.0, 83.3 and $62.8 \%$ for the PP, respectively, for prediction of metastasis to PRLNs (Table III).

Associations of size and nIC of LPLNs for LPLN metastasis. The associations of the maximum short axis diameter of LPLNs and $\mathrm{nIC}$ in the AP and PP in cases with and without LPLN metastasis are presented in Table IV. The median maximum short axis diameter of the LPLNs was significantly larger in LPLN metastasis-positive cases compared with LPLN metastasis-negative cases (9.1 vs. $4.8 \mathrm{~mm}$; $\mathrm{P}=0.03$ ). The median nIC of the maximum-size LPLN was insignificantly different between LPLN metastasis-positive and -negative cases in the AP ( 0.15 vs. $0.21 ; P=0.19)$, but was significantly lower in LPLN metastasis-positive cases compared with LPLN metastasis-negative cases in the PP (0.29 vs. 0.55; $\mathrm{P}=0.04)$. The cut-off values for nICs of LPLNs were 7.0 (AUC, 0.912) and $0.29 \mathrm{~mm}$ (AUC, 0.877) in the AP and PP, respectively (Fig. 3A and $\mathrm{B}$ ), and these cut-off values provided
Table I. Patient characteristics.

\begin{tabular}{|c|c|}
\hline Characteristics & Value \\
\hline Male, n (\%) & $34(77.2)$ \\
\hline Median Age, years (range) & $65(36-82)$ \\
\hline Median BMI, kg/m² (range) & $21.6(16.0-31.0)$ \\
\hline Median distance of tumor from & $5(2-15)$ \\
\hline \multicolumn{2}{|l|}{$\mathrm{AV}, \mathrm{cm}$ (range) } \\
\hline Location of tumor: Rb, P, n (\%) & $33(75)$ \\
\hline \multicolumn{2}{|l|}{ Clinical T stage, $\mathrm{n}(\%)$} \\
\hline 1 (M-SM) & $2(4.5)$ \\
\hline $2(\mathrm{MP})$ & $6(13.6)$ \\
\hline $3(\mathrm{SS}, \mathrm{A})$ & $23(52.3)$ \\
\hline $4(\mathrm{SE}, \mathrm{SI}, \mathrm{AI})$ & $13(29.6)$ \\
\hline \multicolumn{2}{|l|}{ Clinical N stage, n (\%) } \\
\hline 0 & $23(52.3)$ \\
\hline 1 (number of metastatic LNs; $1-3$ ) & $5(11.4)$ \\
\hline 2 (number of metastatic LNs; $\geq 4$ ) & $6(13.6)$ \\
\hline 3 (with LPLN metastasis) & $10(22.7)$ \\
\hline Preoperative chemotherapy, n (\%) & $25(56.8)$ \\
\hline Laparoscopic, robot, n (\%) & $40(90.1)$ \\
\hline Median operation time, min (range) & $294.5(121-487)$ \\
\hline Median blood loss, ml (range) & $50(0-2160)$ \\
\hline pT3, T4, n (\%) & $26(59.1)$ \\
\hline LNND, n (\%) & $24(54.5)$ \\
\hline PRLN metastasis, $\mathrm{n}(\%)$ & $15(34.1)$ \\
\hline LPLN metastasis, $\mathrm{n}(\%)$ & $3(12.5)$ \\
\hline Rate of anal preservation, n (\%) & $33(75)$ \\
\hline
\end{tabular}

BMI, body mass index; AV, anal verge; LNND, lateral lymph node dissection; PRLN, pararectal lymph node; LPLN, lateral pelvic lymph node.

a sensitivity, specificity, PPV, NPV and accuracy of 100, 84.2, 50, 100 and $86.4 \%$ for the AP, and 66.7, 100, 100, 95.2 and $95.7 \%$ for the PP, respectively, for prediction of metastasis to LPLNs (Table V).

\section{Discussion}

The cut-off value of size-based diagnosis for rectal cancer is inconsistent; for example, Akiyoshi et al (5) reported that $8 \mathrm{~mm}$ is the optimum cut-off for prediction of LPLN metastasis on MRI, whereas Ogawa et al (6) proposed a cut-off of $5 \mathrm{~mm}$. Therefore, the accuracy of size-based diagnosis is uncertain and other methods to predict metastasis have been examined. Akiyoshi et al (5) suggested that a mixed signal intensity (a mixture of various intensities) was frequent in cases with LPLN metastasis; however, no significant difference was revealed in multivariate analysis and prediction was based on a subjective qualitative judgment by radiologists so this method required advanced expertise. The nIC on DECT may provide a solution to this problem. DECT uses two tubular bulbs for fast switching and density-based analysis of materials, including iodine, is possible $(14,15)$. In $\mathrm{RC}$, in addition to evaluation of the primary lesion (16), DECT 
Table II. Association between PRLN metastasis and short axis diameter of PRLNs, nIC value of PRLNs.

\begin{tabular}{|c|c|c|c|}
\hline Parameter & PRLN metastasis $(-)(n=29)$ & PRLN metastasis $(+)(n=15)$ & P-value \\
\hline \multicolumn{4}{|c|}{ Median size of PRLN (mm) } \\
\hline Short axis & $6.4(3.4-11.1)$ & $7.6(4.0-17.0)$ & 0.33 \\
\hline \multicolumn{4}{|c|}{ Median nIC value } \\
\hline AP & $0.25(0.10-0.41)$ & $0.18(0.05-0.27)$ & 0.01 \\
\hline $\mathrm{PP}$ & $0.61(0.16-0.96)$ & $0.47(0.17-0.68)$ & 0.03 \\
\hline
\end{tabular}

PRLN, pararectal lymph node; nIC, normalized iodine concentration; AP, arterial phase; PP, portal venous phase.
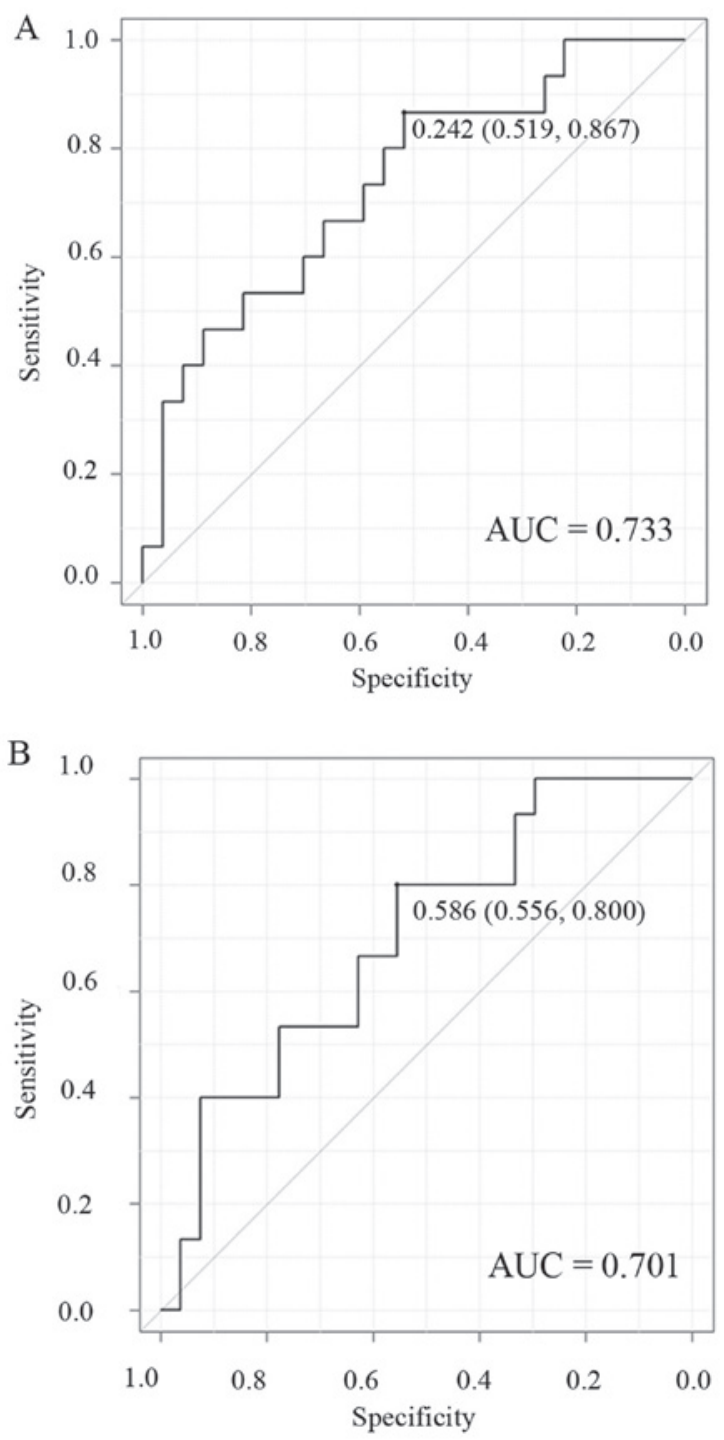

Figure 2. ROC curve analyses for the nIC value in predicting pararectal lymph node metastasis. (A) AUC of the nIC value in the arterial phase (cutoff; 0.242) was 0.733. (B) AUC of the nIC value in the portal venous phase (cutoff; 0.586) was 0.701. ROC, receiver operating characteristic; nIC, normalized iodine concentration; AUC, area under the curve.

has been used to predict PRLN metastasis by Liu et al (7) and Kato et al (17), who identified a significantly lower nIC in pathological metastasis-positive cases compared with negative cases. The nIC was also lower in LN metastasis-positive cases in the current study. Histopathologically, Naresh et al (18) identified
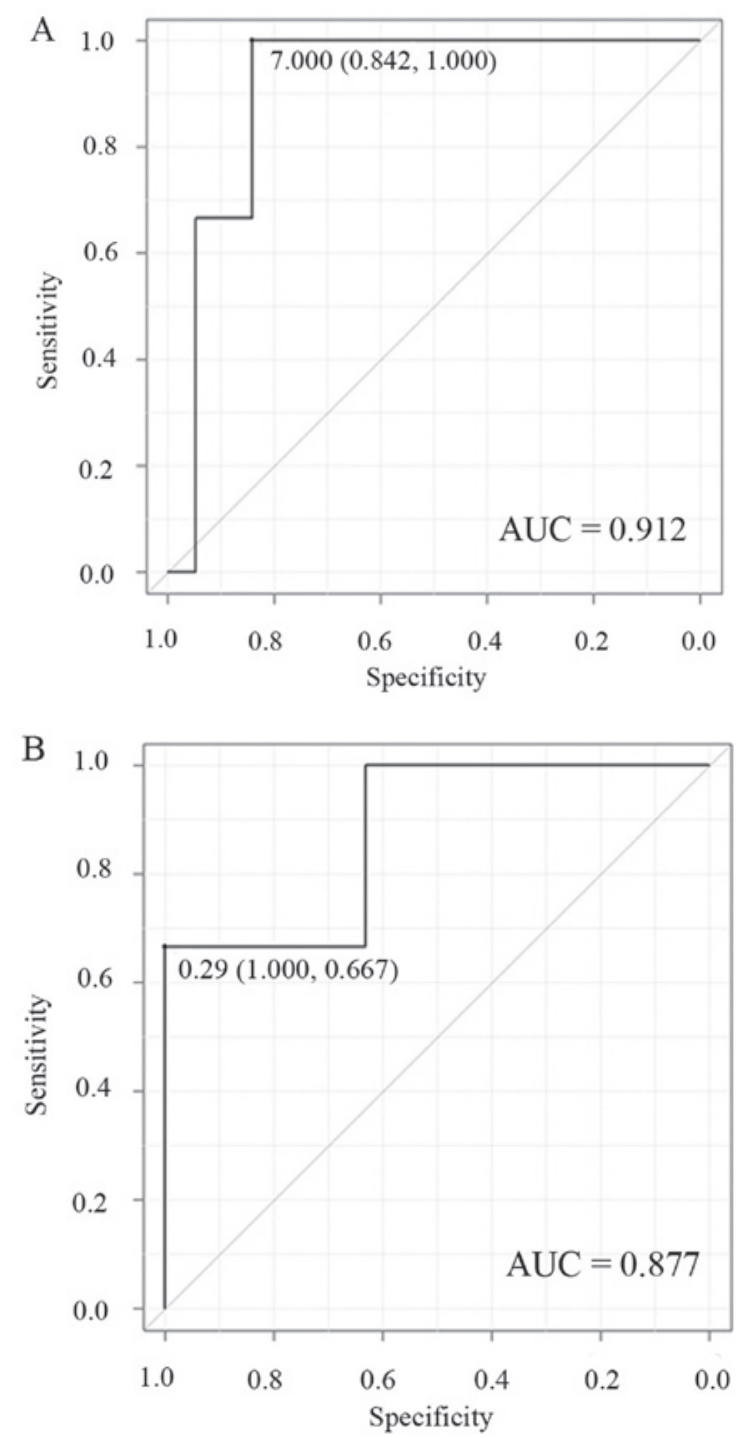

Figure 3. ROC curve analyses for the short axis diameter and nIC value in predicting lateral lymph node metastasis. (A) AUC of the short axis diameter (cutoff; $7.0 \mathrm{~mm}$ ) was 0.912 . (B) AUC of the nIC value in the portal venous phase (cutoff; 0.29) was 0.877. ROC, receiver operating characteristic; nIC, normalized iodine concentration; AUC, area under the curve.

that the number of blood vessels was smaller in metastatic LNs in head and neck cancer, and the nIC on DECT may reflect this pathological feature. Since fewer blood vessels enter metastatic LNs, the nIC may decrease compared with that in non-metastatic lymph nodes. 
Table III. Cut-off value of nIC value in PRLN metastasis and diagnostic performance to PRLN metastasis.

\begin{tabular}{lcccccccc}
\hline Parameter & AUC & 95\% CI & Cutoff & Sensitivity, $\%$ & Specificity, $\%$ & PPV, $\%$ & NPV, $\%$ & Accuracy, $\%$ \\
\hline AP & 0.733 & $0.57-0.89$ & 0.24 & 86.7 & 51.9 & 48.1 & 87.5 & 62.8 \\
PP & 0.701 & $0.54-0.87$ & 0.59 & 80 & 55.6 & 48 & 83.3 & 62.8 \\
\hline
\end{tabular}

nIC, normalized iodine concentration; PRLN, pararectal lymph node; AP, arterial phase; PP, portal phase; AUC, area under the curve; 95\% CI, 95\% confidence interval; PPV, positive predictive value; NPV, negative predictive value.

Table IV. Association between LPLN metastasis and short axis diameter of LPLNs, nIC value of LPLNs.

\begin{tabular}{lccr}
\hline Parameter & LPLN metastasis $(-)(\mathrm{n}=21)$ & LPLN metastasis $(+)(\mathrm{n}=3)$ & P-value \\
\hline $\begin{array}{l}\text { Median size of LPLN }(\mathrm{mm}) \\
\text { Short axis }\end{array}$ & $4.8(3.0-19.5)$ & $9.1(7.0-12.1)$ & 0.03 \\
Median nIC value & & $0.15(0.06-0.21)$ & 0.19 \\
AP & $0.21(0.1-0.32)$ & $0.29(0.23-0.48)$ & 0.04 \\
PP & $0.55(0.32-0.73)$ & & \\
\hline
\end{tabular}

LPLN, lateral pelvic lymph node; nIC, normalized iodine concentration; AP, arterial phase; PP, portal phase.

Table V. Cut-off value of short axis diameter of LPLN and nIC value in LPLN metastasis and diagnostic performance to LPLN metastasis.

\begin{tabular}{|c|c|c|c|c|c|c|c|c|}
\hline Parameter & AUC & $95 \%$ CI & Cutoff & Sensitivity, $\%$ & Specificity, \% & PPV, \% & NPV, \% & Accuracy, $\%$ \\
\hline \multicolumn{9}{|c|}{ Size of LPLN (mm) } \\
\hline Short axis & 0.912 & $0.78-1$ & $7.0 \mathrm{~mm}$ & 100 & 84.2 & 50 & 100 & 86.4 \\
\hline \multicolumn{9}{|l|}{ nIC value } \\
\hline PP & 0.877 & $0.63-1$ & 0.29 & 66.7 & 100 & 100 & 95.2 & 95.7 \\
\hline
\end{tabular}

Disease control by surgery alone is limited for advanced rectal cancer with LN metastasis, and increased preoperative treatment is apparent in recent studies following the prediction of LN metastasis of lower RC. This includes a recent introduction of preoperative chemoradiotherapy, including NACRT, and chemotherapy, including NAC, in Japan (19-21), and a reduction of local recurrence has been reported. Prediction of LN metastasis prior to surgery is important for appropriate use of preoperative treatment. Furthermore, improving the diagnostic reliability of LPLN metastasis is also important to select the patients appropriate for LPLN dissection.

In the current study, the efficiency of DECT was investigated for both PRLNs and LPLNs. For PRLNs, the median maximum short axis diameter of PRLNs was insignificantly different between metastasis-positive and -negative cases; however, the nIC in the AP and PP on DECT was significantly lower for metastatic PRLNs. This suggests that DECT is more useful compared with the size of the LNs for prediction of metastasis. By' contrast, for LPLNs, the maximum short axis diameter of the LNs and nIC in the PP were both useful predictors of metastasis. A cut-off for the maximum short axis of the LNs of $7.0 \mathrm{~mm}$ based on ROC analysis provided an AUC of 0.912 and accuracy of $86.4 \%$, and a cut-off nIC in the PP of 0.29 provided an AUC of 0.877 and accuracy of $95.7 \%$. This suggests that a high preoperative diagnostic accuracy may be obtained using a combination of size-based diagnosis and nIC on DECT for LPLNs. To the best of our knowledge, preoperative prediction of LPLN metastasis by DECT has not been previously reported, and further accumulation and investigation of metastatic LN samples is required. Since numerous PRLNs are dissected, there is likely to be inconsistency between LNs identified on imaging and metastatic LNs, and this may explain the low diagnostic accuracy of DECT for PRLNs compared with that for LPLNs.

The present study had a number of limitations. Firstly, the sample size of 44 patients, including 24 with LPLN dissection, was small and the analysis was retrospective. Only one largest PRLN and LPLN were studied in each case, and it is unclear whether this LN was consistent with the pathological metastasis-positive LN. The probability of inconsistency was high, particularly for PRLNs, as aforementioned. To increase 
the accuracy, a method is required to match the LN identified on DECT with the LN in the resected specimen. Establishing a cut-off value of nIC in a large-scale prospective study using standard measurement methods is also required for clinical application. Within these limitations, it can be concluded that DECT may be useful for preoperative prediction of metastasis to PRLNs and LPLNs. For LPLNs, high diagnostic accuracy may be achieved by combination with size-based diagnosis.

\section{Acknowledgements}

Not applicable.

\section{Funding}

No funding was received.

\section{Availability of data and materials}

All data generated and analyzed in the present study are included in this published article.

\section{Authors' contributions}

KS, HM, FT, YS, TM, HF, KU, TS, ST, RK, SO, MO and KH authors contributed to the conception and design of the study. KS and RK analyzed the images. FT and HF advised the analysis of images. YS, HM, TM, KU, TS and ST performed the surgeries. SO, MA and KH supervised the study. All authors participated in the interpretation of the results and the writing of the manuscript. All authors read and approved the final manuscript.

\section{Ethics approval and consent to participate}

The present retrospective study was approved by the Human Research Ethics Committee of the Hirosaki University Graduate School of Medicine (approval no. 2018-1047).

\section{Patient consent for publication}

Consent was obtained from the patients, who had the option to withdraw from the present study.

\section{Competing interests}

The authors declare that they have no competing interests.

\section{References}

1. Watanabe T, Itabashi M, Shimada Y, Tanaka S, Ito Y, Ajioka Y, Hamaguchi T, Hyodo I, Igarashi M, Ishida H, et al: Japanese society for cancer of the colon and rectum (JSCCR) guidelines 2014 for treatment of colorectal cancer. Int J Clin Oncol 20: 207-239, 2015.

2. MERCURY Study Group, Shihab OC, Taylor F, Bees N, Blake H, Jeyadevan N, Bleehen R, Blomqvist L, Creagh M, George C, et al: Relevance of magnetic resonance imaging detected pelvic sidewall lymph node involvement in rectal cancer. Br J Surg 98: 1798-1804, 2011.

3. Bosset JF, Collette L, Calais G, Mineur L, Maingon P, Radosevic-Jelic L, Daban A, Bardet E, Beny A and Ollier JC: EORTC radiotherapy group trial 22921: Chemotherapy with preoperative radiotherapy in rectal cancer. NEngl J Medicine 355: 1114-1123, 2006.
4. Schrag D, Weiser MR, Goodman KA, Gonen M, Hollywood E, Cercek A, Reidy-Lagunes DL, Gollub MJ, Shia J, Guillem JG, et al: Neoadjuvant chemotherapy without routine use of radiation therapy for patients with locally advanced rectal cancer: A pilot trial. J Clin Oncol 32: 513-518, 2014.

5. Akiyoshi T, Matsueda K, Hiratsuka M, Unno T, Nagata J, Nagasaki T, Konishi T, Fujimoto Y, Nagayama S, Fukunaga Y and Ueno M: Indications for lateral pelvic lymph node dissection based on magnetic resonance imaging before and after preoperative chemoradiotherapy in patients with advanced low-rectal cancer. Ann Surg Oncol 3 (Suppl 22): S614-S620, 2015.

6. Ogawa S, Hida JI, Ike H, Kinugasa T, Ota M, Shinto E, Itabashi M, Okamoto T, Yamamoto M, Sugihara K and Watanabe T: Prediction of lateral pelvic lymph node metastasis from lower rectal cancer using magnetic resonance imaging and risk factors for metastasis: Multicenter study of the lymph node committee of the japanese society for cancer of the colon and rectum. Int J Colorectal Dis 32: 1479-1487, 2017.

7. Liu H, Yan F, Pan Z, Lin X, Luo X, Shi C, Chen X, Wang B and Zhang H: Evaluation of dual energy spectral CT in differentiating metastatic from non-metastatic lymph nodes in rectal cancer: Initial experience. Eur J Radiol 84: 228-234, 2015.

8. Fujita S, Mizusawa J, Kanemitsu Y, Ito M, Kinugasa Y, Komori K, Ohue M, Ota M, Akazai Y, Shiozawa M, et al: Mesorectal excision with or without lateral lymph node dissection for clinical stage II/III lower rectal cancer (JCOG0212): A multicenter, randomized controlled, noninferiority trial. Ann Surg 266: 201-207, 2017.

9. Kobayashi H, Mochizuki H, Kato T, Mori T, Kameoka S, Shirouzu K and Sugihara K: Outcomes of surgery alone for lower rectal cancer with and without pelvic sidewall dissection. Dis Colon Rectum 52: 567-576, 2009.

10. Akasu T, Sugihara K and Moriya Y: Male urinary and sexual functions after mesorectal excision alone or in combination with extended lateral pelvic lymph node dissection for rectal cancer. Ann Surg Oncol 16: 2779-2786, 2009.

11. Japanese Society for Cancer of the Colon and Rectum: Japanese Classification of Colorectal Carcinoma. 8th Edition. Kanehara Shuppan, Tokyo, Japan, 2013.

12. Aoki M, Takai Y, Narita Y, Hirose K, Sato M, Akimoto H, Kawaguchi H, Hatayama Y, Miura H and Ono S: Correlation between tumor size and blood volume in lung tumors: A prospective study on dual energy gemstone spectral CT imaging. J Radiat Res 55: 917-923, 2014.

13. Kanda Y: Investigation of the freely available easy-to-use software 'EZR' for medical statistics. Bone Marrow Transplantat 48: 452-458, 2013.

14. Matsumoto K, Jinzaki M, Tanami Y, Ueno A, Yamada M and Kuribayashi S: Virtual monochromatic spectral imaging with fast kilovoltage switching: Improved image quality as compared with that obtained with conventional $120-\mathrm{kVp}$ CT. Radiology 259: 257-262, 2011.

15. Zhang D, Li X and Litl B: Objective characterization of GE discovery CT750 HD scanner: Gemstone spectral imaging mode. Med Phys 38: 1178-1188, 2011.

16. Morohashi H, Sakamoto Y, Ichinohe D, Jin H, Miura T, Tsushima F Ono S and Hakamada K: Evaluation of the therapeutic effect of using dual-energy CT for rectal cancer after neoadjuvant chemotherapy. Gan To Kagaku Ryoho 43: 1482-1484, 2016 (In Japanese).

17. Kato T, Uehara K, Ishigaki S, Nihashi T, Arimoto A, Nakamura H, Kamiya T, Oshiro T, Ebata T and Nagino M: Clinical significance of dual-energy CT-derived iodine quantification in the diagnosis of metastatic LN in colorectal cancer. Eur J Surg Oncol 41: 1464-1470, 2015.

18. Naresh KN, Nerurkar AY and Borges AM: Angiogenesis is redundant for tumour growth in lymph node metastases. Histopathology 38: 466-470, 2001.

19. Kamiya T, Uehara K, Nakayama G, Ishigure K, Kobayashi S, Hiramatsu K, Nakayama H, Yamashita K, Sakamoto E, Tojima Y, et al: Early results of multicenter phase II trial of perioperative oxaliplatin and capecitabine without radiotherapy for high-risk rectal cancer: CORONA I study. Eur J Surg Oncol 42: 829-835, 2016.

20. Hasegawa S, Goto S, Matsumoto T, Hida K, Kawada K, Matsusue R, Yamaguchi T, Nishitai R, Manaka D, Kato S, et al: A multicenter phase 2 study on the feasibility and efficacy of neoadjuvant chemotherapy without radiotherapy for locally advanced rectal cancer. Ann Surg Oncol 24: 3587-3595, 2017.

21. Nakamura T, Yamashita K, Sato T, Ema A, Naito M and Watanabe M: Neoadjuvant chemoradiotherapy using S-1 and irinotecan in rectal cancer: Impact on long-term clinical outcomes and prognostic factors. Int J Radiat Oncol Biol Phys 89: 547-555, 2014. 